\title{
Vorwort zur elften Auflage
}

Die zehnte Auflage ist innerhalb eines Jahres vergriffen worden. Auch in der elften Auflage habe ich mich bemüht, das Buch durch Nachträge und Ergänzungen auf den neuesten Stand zu bringen.

Köln, 20. Mai $19 \check{8} 8$

Heinrich Lehmann

\section{Vorwort zur fünften Auflage}

Der Allgemeine Teil des Bürgerlichen Gesetzbuches ist wieder in seine alten Rechte eingesetzt worden. Damit werden die Wünsche aller derer erfüllt, die das Bedürfnis empfinden, die Fülle des privatrechtlichen Stoffes von einem höheren Blickpunkt aus zu übersehen, die leitenden Grundgedanken herauszuarbeiten und zu einem einheitlichen Bau zu ordnen - also namentlich die Wünsche der Studenten, die bei dem Rechtsstudium nach der aufgehobenen nazistischen Studienordnung über mangelnden Ưberblick klagten, aber auch die Wünsche der fertigen Juristen, denen die Rechtsanwendung mehr als eine handwerksmäßige Fertigkeit in der Handhabe der einzelnen Gesetzesbestimmungen bedeutet.

Die Bedenken, die man in dem letzten Jahrzwölft gegen den Allgemeinen Teil geltend gemacht hat, lassen sich keinenfalls gegen die wissenschaftliche Herausarbeitung der Grundsätze erheben, die ein Rechtsgebiet beherrschen, sondern allenfalls gegen die gesetzliche Festlegung solcher Grundsätze durch vorzeitig verallgemeinerte Begriffe, also ehe das gesamte Tatsachenmaterial, für das sie in Betracht kommen, klar angeschaut und abgegrenzt ist. Das ist allerdings im Kernstück des Allgemeinen Teils, dem Abschnitt über Rechtsgeschäfte (\$\$ 104-185 BGB.), nicht überall hinreichend beachtet worden. Es ist eine Schwäche des Allgemeinen Teils, daß seine Vorschriften über die Rechtsgeschäfte, obwohl sie z. T. nur Abstraktionen aus den angeschauten Tatbeständen des individualrechtlichen Verkehrs unter Einzelpersonen sind, gleichwohl ihrem Wortlaut nach allgemeine An- 
forderungen für alle Rechtsgeschäfte enthalten. So hat sich in den verflossenen Jahrzehnten immer mehr herausgestellt, dab diese Vorschriften der Eigenart der sozialrechtlichen Geschäfte, wie z. B. der des Verlöbnisses, der Gesellschaftsgründung, der Beitrittserklärung zur Gesellschaft, Beschluß und Abstimmung usw. nicht hinreichend gerecht werden. Das hat den Nachteil mit sich gebracht, daB ein vom Gesetzgeber in seiner Wesensart noch nicht richtig gewerteter sozialrechtlicher Tatbestand unter eine nicht passende Vorschrift gebracht wurde. Das wurde besonders bei der Einkleidung des Verlöbnisses in die Form eines ,Vertrages" im Sinne des Allgemeinen Teils durch das Reichsgericht (RG.61 270 u. 80 89) klar. Der Rechtsprechung fällt es erfahrungsgemäß viel schwerer, Restriktion zu treiben als sich zur Analogie zu entschließen, obwohl jene als ein Engerdenken der Norm aus dem vernünftigen Gesetzeszweck heraus genauso berechtigt und notwendig ist als diese, die den im Gesetz niedergelegten Grundgedanken für die nicht geregelten, aber gleich zu behandelnden Tatbestände zu Ende denkt.

Die mit den Abstraktionen des Allgemeinen Teils verbundene Gefahr läßt sich durch eine richtige Gesetzes anwendung mit diesen beiden Mitteln durchaus bannen, wenn die Rechtsprechung der Aufgabe gerecht wird, jeder Anwendung einer gesetzlichen Vorschrift eine sorgfältige Untersuchung der durch sie getroffenen Tatsachen und Lebensverhältnisse sowie des vernünftig begrenzten oder erstrebten Gesetzeszweckes vorangehen zu lassen. Das Gesetz will im Zweifel die vernünftige und gerechte Ordnung der Lebensverhältnisse evident machen, nicht aber diese unpassend zurechtstutzen.

In diesem Sinne verstanden und gehandhabt, verlieren die Vorschriften des Allgemeinen Teils das Bedenkliche zu weit getriebener Abstraktion. Statt ein Prokrustesbett der Lebenstatbestände werden sie ein unvergleichliches Mittel zur Beherrschung, systematischen Ordnung und gerechten Handhabung der positiven Rechtsnormen.

Köln, Weihnachten 1946 